\title{
Discontinuation of a randomised controlled trial in general practice due to unsuccessful patient recruitment
}

\author{
Wendelien $\mathrm{H}$ van der Gaag, MD ${ }^{1 *}$, Roxanne van den Berg, $\mathrm{MD}^{2}$, \\ Bart W Koes, $\mathrm{PhD}^{3}$, Arthur M Bohnen, MD, $\mathrm{PhD}^{4}$, Lonny MG Hazen, $\mathrm{MD}^{5}$, \\ Wilco C Peul, MD, PhD ${ }^{6}$, Leen Voogt ${ }^{7}$, Arianne $\mathrm{P}$ Verhagen, $\mathrm{PhD}^{8}$, \\ Sita MA Bierma-Zeinstra, PhD ${ }^{9}$, Pim AJ Luijsterburg, PhD ${ }^{10}$
}

${ }^{1} \mathrm{GP}$ trainee and PhD student, Department of General Practice, Erasmus MC, University Medical Center, Rotterdam, The Netherlands; ${ }^{2} \mathrm{GP}$ trainee and $\mathrm{PhD}$ student, Department of General Practice, Erasmus MC, University Medical Center, Rotterdam, The Netherlands; ${ }^{3}$ Professor of General Practice, Department of General Practice, Erasmus MC, University Medical Center, Rotterdam, The Netherlands; ${ }^{4} \mathrm{GP}$, Department of General Practice, Erasmus MC, University Medical Center, Rotterdam, The Netherlands; ${ }^{5} \mathrm{GP}$, Department of General Practice, Erasmus MC, University Medical Center, Rotterdam, The Netherlands; ' ${ }^{6}$ Professor of Neurosurgery, Department of Neurosurgery, Leiden University Medical Center, Leiden, The Netherlands; ${ }^{7}$ Patient and Board Member of 'De Wervelkolom', Dutch Association for Back Pain Patients, Lichtenvoorde, The Netherlands; ${ }^{8}$ Associate Professor, Department of General Practice, Erasmus MC, University Medical Center, Rotterdam, The Netherlands; ${ }^{9}$ Professor of Osteoarthritis and Related Disorders, Departments of General Practice and Orthopedics, Erasmus MC, University Medical Center, Rotterdam, The Netherlands; ${ }^{10}$ Assistant Professor, Department of General Practice, Erasmus MC, University Medical Center, Rotterdam, The Netherlands

*For correspondence: w.vandergaag@erasmusmc.nl

Competing interests: The authors declare that no competing interests exist.

Received: 23 December 2016 Accepted: 27 March 2017 Published: 23 August 2017

(c) This article is Open Access: CC BY license (https:// creativecommons.org/licenses/ by/4.0/)

Author Keywords: primary health care, general practice, randomized controlled trial, patient recruitment, early termination of clinical trials, study design

Copyright (C) The Authors 2017; DOI:10.3399/

bjgpopen17X101085

\section{Abstract}

Background: A randomised controlled trial (RCT) in general practice, recruiting incident patients with (sub)acute sciatica, was discontinued because of insufficient recruitment.

Aim: To describe factors that influenced the recruitment process and ultimately led to discontinuation of this trial, and to enable others to learn from this experience.

Design \& setting: A pragmatic RCT was designed to compare two pain medication prescription strategies for treatment of (sub)acute sciatica in general practice. After 1 year of patient recruitment, the trial was prematurely terminated.

Method: To analyse the underperforming recruitment, patient information systems of 20 general practices were screened twice a month to search for eligible patients and identify reasons for noneligibility. Secondly, after study termination, an open question was distributed to the participating GPs for their views on the recruitment process.

Results: A total of 116 GPs from 37 general practices collaborated in the trial. Only eight of 234 patients were included after 12 months. The 22 GPs who offered their opinion on the main reasons for unsuccessful recruitment considered that these were the low incidence rate and strict eligibility criteria, a strong patient and/or GP preference, and time constraints.

Conclusion: For this RCT, multiple factors were related to recruitment problems but it remains unknown which determinants prevailed. As the research question is unanswered but remains relevant, it is recommended that GPs' daily practice is taken into account when designing an RCT, a 
pilot study should be performed for feasibility of recruitment, and GP assistants should be involved at an early stage.

\section{How this fits in}

Research in the field of general practice is essential to expand evidence for guidelines in the diagnosis and treatment of diseases within primary care. However, in practice, study protocols are not always congruent with the reality in general practice. This article describes the rationale behind a randomised controlled trial that was prematurely discontinued due to insufficient patient recruitment. The following recommendations for future research are to design convenient protocols that facilitate referral to research as a routine task for GPs, with the least possible interference with daily regular practice, to perform a pilot study, and to outsource eligibility screening to trained GP assistants or dedicated research nurses.

\section{Introduction}

General practice has changed rapidly in recent decades. Ageing populations and increasing care for patients with chronic diseases and (complex) multimorbidity demand expanding service profiles. ${ }^{1}$ These changing health needs have shaped the developments in general practice in the Netherlands and elsewhere. For example, both patient list size per GP and the average patient contact rate with the general practice have increased. ${ }^{2-4}$ Dutch GPs spend (on average) more time on non-patient bound activities (such as administration, management, and continuing vocational training) than their European counterparts. ${ }^{1,5}$ Task delegation to practice assistants or nurses is inevitable and has become regular practice. The question then arises whether conducting clinical research is more difficult in today's general practices, due to changes in priority setting; that is, changes that might now be inevitable to keep the workload manageable.

For evidence-based decision making in general practice, clinical research is essential and most GPs are aware of this. ${ }^{6,7}$ Researchers, on the other hand, have to translate their research questions and ideas into concrete trial designs that are feasible in general practice. The design needs methodological soundness and adequate power to answer the research questions. ${ }^{8,9} \mathrm{~A}$ complicating factor is that the protocol must have minor interference with daily practice to be feasible. ${ }^{10}$ Thus, a delicate balance is required between patients and GPs' needs, and research aims that will ultimately benefit the patient.

The authors developed and received funding for a pragmatic design of an RCT on sciatica, the most prevalent specific type of low back disorders seen by GPs. Current treatment of sciatica in general practice aims to enable patients to stay active and return to daily activities as soon as possible. ${ }^{11}$ Therefore, pain relief through adequate analgesic(s) prescription is an important condition; however, the best approach for prescription of pain medication in sciatica remains unclear. $^{12}$ This RCT, the STEP-UP trial, aimed to assess the clinical and cost-effectiveness of immediate opioid pain medication (followed by step-down) versus stepped-up pain medication according to the current clinical GP guidelines, in patients with (sub)acute sciatica in general practice over a period of 12 weeks. $^{13}$

During 12 months of recruiting in general practices, 23 patients were assessed for eligibility by the research team, of which only eight (3.4\% of the target 234 patients) could be included in the trial. Because the inclusion rate was too slow to recruit a sufficient number of patients in the remaining time available, the study was prematurely terminated after 1 year.

The aim of this study was to describe determinants in the recruitment process that ultimately led to discontinuation of this trial.

\section{Method}

\section{Trial design and setting}

The STEP-UP trial was a pragmatic, multicentre, open-label, superiority RCT with a parallel group design, in general practices, with a 3-month follow-up, as shown in Box 1 and Figure $1 .^{13}$ Patients consulting their GP with (sub)acute sciatica were randomly allocated to receive stepped- 
Box 1. STEP-UP trial design

\begin{tabular}{|c|c|}
\hline Design & $\begin{array}{l}\text { A pragmatic, multicentre, open-label, randomised controlled trial with parallel group design, in } \\
\text { general practices with a } 3 \text {-month follow-up period }\end{array}$ \\
\hline Setting & General practices in the southwestern area of the Netherlands \\
\hline Objective & $\begin{array}{l}\text { To assess the clinical and cost-effectiveness of two pain medication prescription strategies in } \\
\text { general practice over a 12-week period }\end{array}$ \\
\hline $\begin{array}{l}\text { Eligibility } \\
\text { criteria }\end{array}$ & $\begin{array}{l}\text { Inclusion criteria } \\
\text { 1. Age } 18-65 \text { years } \\
\text { 2. No use of opioids } \\
\text { 3. Radiating (pain) complaints in one leg below the knee } \\
\text { 4. Severity of radiating leg pain scored } \geq 7 \text { on an } 11 \text {-point numerical rating scale }(0= \\
\text { 'no pain'; } 10=\text { 'worst pain imaginable') } \\
\text { 5. Duration of the (pain) complaints }<12 \text { weeks } \\
\text { 6. Presence of at least one of the following symptoms: } \\
\text { 1. More pain on coughing, sneezing, or straining } \\
\text { 2. Decreased muscle strength in the leg } \\
\text { 3. Sensory deficits in the leg } \\
\text { 4. Decreased reflex activity in the leg } \\
\text { 5. Positive straight leg raising test }\end{array}$ \\
\hline & $\begin{array}{l}\text { Exclusion criteria } \\
\text { 1. An episode of radiating (pain) complaints in the preceding } 6 \text { months } \\
\text { 2. Back surgery in the past } 3 \text { years } \\
\text { 3. Treated with epidural injections } \\
\text { 4. Pregnancy } \\
\text { 5. Comorbidity that primary determines overall wellbeing (for example, an osteoporotic } \\
\text { fracture, malignity, herpes zoster, or Lyme's disease) } \\
\text { 6. Hypersensitivity to paracetamol, NSAID, or opioids } \\
\text { 7. Previous or active peptic ulcer } \\
\text { 8. Direct indication for surgery (fast progression of paresis of cauda equine syndrome) } \\
\text { 9. History of substance addiction or abuse }\end{array}$ \\
\hline Outcome & $\begin{array}{l}\text { Primary outcome was the severity of radiating leg pain measured daily over a 6-week follow-up } \\
\text { period using an 11-point numerical rating scale (score range 0-10), with a higher score } \\
\text { indicating more pain. }\end{array}$ \\
\hline
\end{tabular}

up medication (step 1: paracetamol; step 2: non-steroidal anti-inflammatory drug [NSAID]; step 3: tramadol; step 4: morphine) or receive immediate morphine; followed by a taper period (step-down).

\section{Selection and recruitment of GP practices}

It was calculated that the cooperation of at least 91 GPs was needed to recruit 234 patients with (sub)acute sciatica within 21 months. ${ }^{14} \mathrm{GP}$ practices were identified in the southwestern area of the Netherlands - selected by zip code - and recruited by mail, followed by a phone call to make an appointment (practice visit or by phone with the choice up to GP) to explain more about the trial. The search area was expanded every month until the numbers were reached; and GP recruitment was continued when it was discovered that patient recruitment from the 91 GPs was behind schedule. A monthly newsletter was sent to update the GPs about the trial, and on request an e-mail reminder was sent every 2 weeks, containing information on eligibility criteria, how to invite patients to join and the consent procedure. Furthermore most general practices were visited regularly to remind GPs and practice nurses about the trial. 


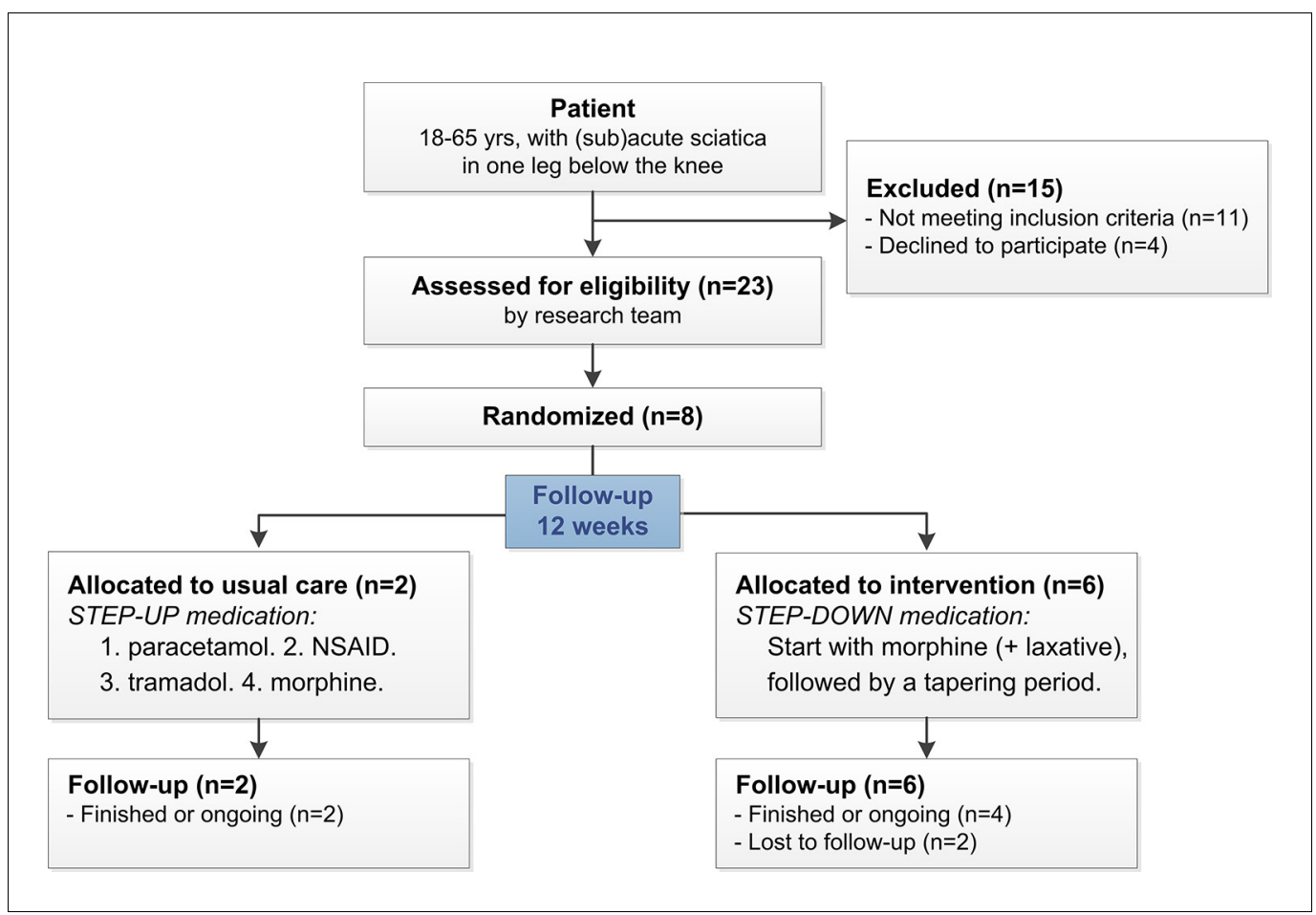

Figure 1. Flow diagram of the STEP-UP trial (pragmatic design RCT).

\section{Patient recruitment process}

Patients were identified in two ways:

1. during GP consultations (1 April 201-30 March 2016), and

2. screening of GP information systems (29 September 2015-4 January 2016).

\section{During the consultations}

The GP performed physical examination, briefly informed eligible patients about the trial, invited them to participate and then notified the research team. The researcher contacted the patient to further assess eligibility, explain the informed consent procedure, and elaborate on any questions. Patients could consider participation in the study for as long as they wished, unless they were not using stronger medication than NSAIDs. After enrolment and randomisation by the research team, both patients and GPs were informed promptly by phone: the GP prescribed medication accordingly and patients could collect the medication the same day. Using this strategy, by September 2015 (after 6 months of recruiting) only one patient was included in the trial.

\section{Screening of GP information systems}

The GP information systems of 20 of the participating general practices (a total of 72 GPs) were screened systematically every 2 weeks during 3.5 months for patients that visited the general practice with International Classification of Primary Care (ICPC) code L86 (sciatica). The GP practices decided for themselves if they would participate in this screening option. If so, every 2 weeks they could assess the patients on the list for eligibility and send letters to invite them for study participation. After a week a reminder letter was sent. Both letters contained the patient information form, a response form, and a stamped return envelope. If patients returned the response form, they were called by the research team to further inform them about the trial, re-assess eligibility and for the informed consent procedure. After enrolment and randomisation by the research team, both 
patients and GPs were informed promptly by phone: the GP prescribed medication accordingly and patients could collect the medication the same day.

\section{Delayed inclusion and discontinuation of the study}

When inclusion was clearly delayed, the problems were discussed during a GP network meeting in September 2015, with about 11 representatives of the participating general practices. Ten GPs were interviewed at their workplace and a research team meeting was scheduled to discuss the main reasons for delay and possible steps to be taken. A STEP-UP month was organised, where all participating GPs and practice nurses were visited to motivate them, strengthen their involvement, and enhance recruitment. A weekly newsletter was sent out to activate GPs and there was a voucher reward for every new patient admitted by a general practice.

Nevertheless, the study was terminated as the measures taken showed insufficient effect. After discontinuation (30 March 2016), each participating GP was sent a written survey, asking: Why do you think we were unable to include a sufficient number of patients in the STEP-UP trial?

\section{Results}

\section{GP recruitment}

GP recruitment started in January 2015 and patient recruitment in April 2015 with the cooperation of eight general practices and 36 GPs. The research question was considered relevant by the GPs that wanted to participate. For the negative responders, the main reasons for declining participation were lack of time, participation in other trials, or lack of interest in the topic. When patient recruitment was discontinued in March 2016, more than 450 general practices and GPs had been invited to join the trial; of these, 37 general practices with 116 GPs were participating in the study.

\section{Patient recruitment}

Figure 2 shows the results of the systematic screening of the GP information systems in 20 general practices. Of the 582 patients screened during the 3.5-month time frame, 71 (12\%) were potentially eligible. GPs sent invitations to 41 of these patients (58\%) of whom 10 (24\%) returned the registration form. Finally, of these, two patients were included and eight were either excluded or declined to participate.

\section{Reasons for insufficient recruitment}

Following the project group meetings and interviews with GPs, factors that could have contributed to the low inclusion were identified.

1. Strict eligibility criteria: based on data from an earlier completed RCT (135 patients with [sub] acute sciatica recruited from 61 GPs over 18 months; May 2003-November 2004), ${ }^{14}$ an estimation was made of the number of GPs needed to recruit 234 patients within 21 months. However, for the present trial the earlier inclusion/exclusion criteria were adapted (that is, they were stricter). For example, in this study, a pain score (visual analogue scale) of $\geq 7$ instead of $\geq 4$ was required to participate, and criteria to prevent adverse events or abuse were added because this study involved NSAIDs and morphine. The effect of these adaptations on recruitment was greater than expected. In retrospect, a more conservative estimation of the speed of recruitment was needed.

2. Incident case study: the trial was designed to enrol patients consulting their GP with (sub)acute sciatica. Inclusion estimates were based on the incidence of sciatica in the Netherlands, which is estimated at 5 per 1000 persons/year in general practice. ${ }^{15}$ In addition, the incidence (all ages) of ICPC code L86 (sciatica) in general practice was 15.2 per 1000 patients/year in 2014, according to the NIVEL Primary Care Database. ${ }^{16}$ However, in fact, due to the restrictive eligibility criteria, only $12 \%$ of the patients with ICPC code L86 were eligible for participation, which results in approximately four eligible patients/year per full-time GP.

Of the 116 GPs, 22 (19\%) responded to the written survey (that is one question about the reasons for recruitment failure) sent to each GP after discontinuation of the study. Their answers are 


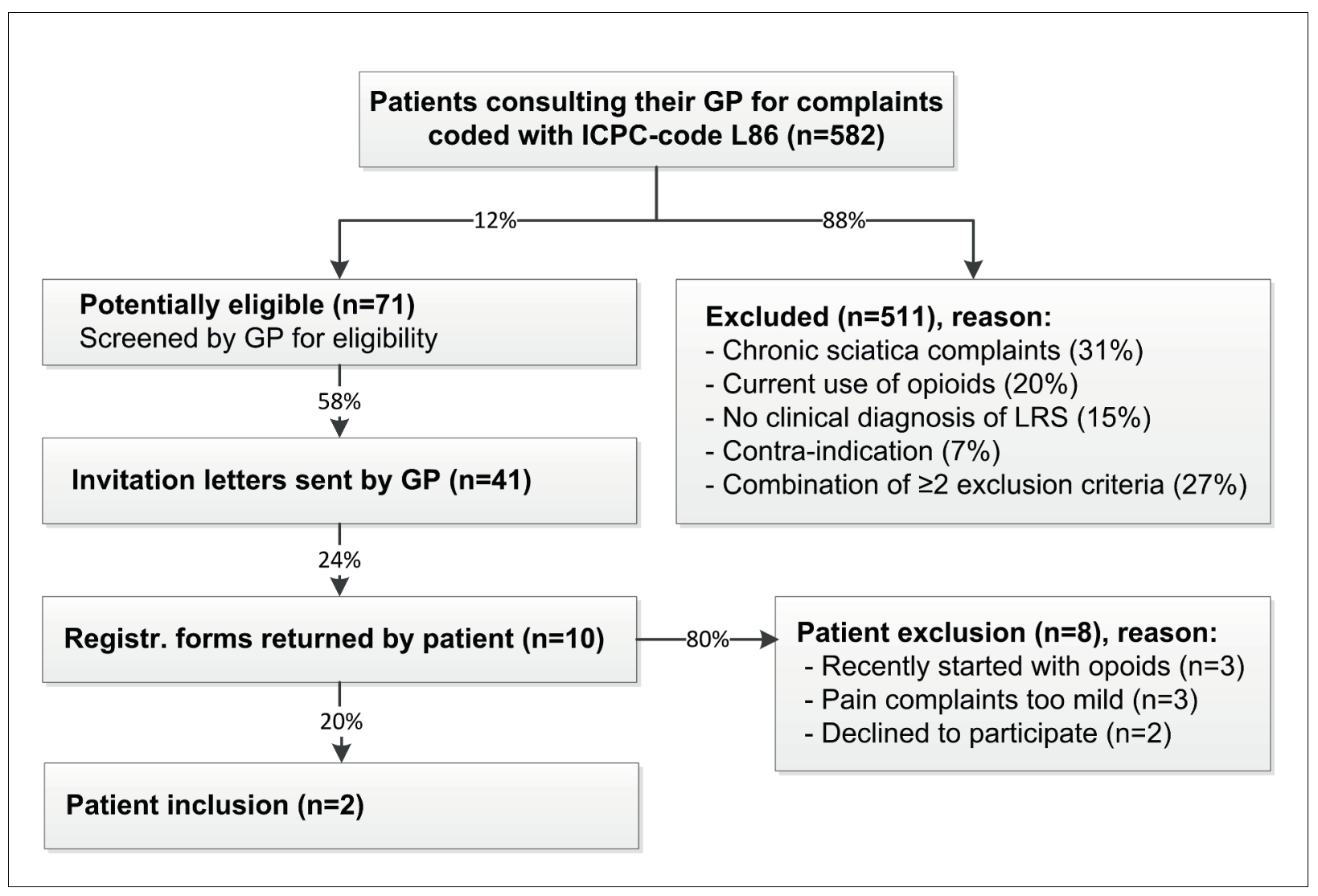

Figure 2. Systematic screening of GP information systems during a 3.5-month period. LRS = lumbosacral radicular syndrome.

presented in Box 2: 11 (50\%) mentioned a strong patient preference and/or GP preference for either the step-up or step-down approach; six GPs (27\%) mentioned the low incidence rate; and six (27\%) the strict eligibility criteria. Other constraints included time and priority constraints, forgetfulness, and prevention of (further) treatment delay for their patients. A summary of reasons is shown in Table 1.

In (unstructured) interviews GPs mentioned a few trends in general practice that also may have influenced the course of this trial:

1. Changes in patient populations and prescription habits: epidemiological research shows a tendency of increased production and utilisation of prescription opioid analgesics for the treatment of non-cancer pain in industrialised countries; this might also apply to our targeted study population. ${ }^{17-21}$ Furthermore, with increasing comorbidity and an ageing population, the use of NSAIDs and/or tramadol is contraindicated more often, which could accelerate the process from step 1 to 4 , by skipping steps $2 / 3$.

2. Process of shared decision making and patient-centred care: the decision-making process in modern general practice has become more patient-centred. The doctor-patient relationship is increasingly based on shared decision making, with respect for patients' autonomy, instead of paternalism. The chosen approach largely depends on patient characteristics and their preference and expectations; ${ }^{22,23}$ for example, for pain medication, personal preferences can be strong. Some GPs mentioned that patients prefer drugs other than morphine, because morphine is considered to be too strong (for example, for fear of adverse events, or wanting to keep driving). Other patients, in contrast, request stronger pain medication and/or diagnostics. Thus, their GPs already have to negotiate to prevent unnecessary diagnostics. Consequently, patients on both sides of the spectrum did not want to be randomised.

3. Priority setting: the changes in both healthcare system and healthcare include societal developments, the transition of several disease treatments and general psychological care from secondary to primary care, decentralisation of youth and elderly care, implementation of new 


\section{Box 2. Responses of 22/116 (19\%) GPs suggested reasons for insufficient recruitment}

1 - Lack of patients who fulfilled eligibility criteria.

- Most patients that considered participation didn't want to use morphine.

- A few patients with a language barrier or contra-indication for the use of either morphine or NSAIDs.

- If, after this, patients could still be included, it was hard to include them and was too demanding in time and effort from the GP.

- As a GP I am already used to prescribe morphine in case of severe pain.

- In the trial design phase these problems were mentioned by GPs; for example in the GP network meeting, but I have the feeling this wasn't taken into account enough.

2 The threshold for patients (and doctor) is (too) high when it comes to prescribing morphine for pain relief. It takes a lot of effort from us as GPs to use diagnostics and titrate patients to morphine: usually this takes 1-2 patient contacts a week. A referral to the neurologist is faster. The low response is a pity: in my opinion it is a very relevant research question.

3 My suspicion is that the researchers overestimated the incidence from ICPC L86. Maybe GPs encode inaccurately. Speaking for myself: when in doubt, if I can choose between L02, L03 or L86, I prefer L03 or L02. L86 seems to have much more consequences; as a GP I prefer some supporting diagnostics to code L86 with certainty. I consider the physical examination alone too limited to do so.

4 - It is hard to remember the trial at the right moment.

- What would help? A financial reward for the GP for admitting a patient to the trial. It's a pity, but that is the case. With $€ 50$ per patient you would see an increase in trial participants.

5 - Low amount of eligible patients.

- Because of this, I don't think about including a patient in the trial at the right moment; I forget it.

6 When the trial was presented, we thought the research question to be very relevant. From the start of the trial I realised I found it hard to leave the choice for pain medication out of hands. In two cases with approximately the same amount of pain, you still choose a different pain management method per patient, depending on many other factors than just the pain. I didn't want 'the randomisation' to decide this. But it's a pity that this means we will not have an answer to the research question.

7 It is difficult to ask a patient to participate in research before starting an intervention (for example, start of medication). So in this case it also didn't help to select patients later with the screening, most of them were already using medication. And for ... research: the GP 'just' forgets about it. The GP assistant is the first contact moment with the patient; it would already help if they ... put a note in the patient's file about the possibility of participating in the trial.

8

Too many research projects at the same time!

9 - Low incidence of the disease. In 12 months I didn't see any new patient with sciatica.

- Not enough time in daily practice for informed consent.

- For screening the patient information system for prevalent cases there is no binding contract concerning patient privacy for the research assistant.

10 - Research topic not that interesting

- Opioid pain medication often has a lot of side effects that are often underestimated. They made a phrase for this for a reason: opioid rotation. Well, and then a lot of patients die after a little while.

- Low frequency of patients with sciatica.

- Too many research projects.

11 - Probably less 'feeling' with the research question.

- I had the experience that a patient I submitted was excluded for the trial.

- Patients often didn't want to participate.

- Later in the trial I lost my motivation.

12 - The pain score threshold for inclusion in the trial was very high (inherent to the possibility of opioid medication as painkillers): that is just quite rare.

- And secondly: in such pain, patients have often already tried a lot of pain medication themselves, which makes participation in a trial and randomisation to the control arm feel like starting over with something you already knew it didn't help.

13 The trial concerns a group of patients that demands and needs direct help. The research setting then causes an unnecessary and unwelcome delay.
- Eligibility criteria

- Shared decision making

- Priority setting

- Other

- Shared decision making

- Priority setting

- Incidence

- Forgetfulness

- Motivation

- Eligibility criteria

- Forgetfulness

- Shared decision making

- Shared decision making

- Delay

- Forgetfulness

- Motivation

- Priority setting

- Incidence

- Priority setting

- Other

- Incidence

- Shared decision making

- Motivation

- Priority setting

- Shared decision making

- Motivation

- Shared decision making

- Eligibility criteria

- Shared decision making

- Delay

continued on next page 
continued

Number Written responses of the 22 GPs

Summary of reasons

14 Incidence of the disease in general practice is too low.

- Incidence

15 - Forgetfulness about the study before inclusion.

- Our general practice joined the trial in January 2016 only.

- The inclusion criteria were very specific and narrow; especially concerning the pain score.

- Eligibility criteria

- Forgetfulness

- Other

16 - Sciatica is apparently less prevalent than expected.

- Or maybe patients go to the physiotherapist by themselves and they don't visit their GP anymore for this complaint? [Note: since 2005 a GP referral is no longer needed to consult a physiotherapist; everyone in the Netherlands is allowed direct access]

17 Very limited number of patients that presented themselves for consultation with sciatica complaints; and then additionally were also eligible for participation in the trial.

- Incidence

- Other

Attention in our general practice is mostly claimed by countless organisational tasks and duties like accreditation, new legislations (recently two new laws) and so on. We can't handle more than sporadic and ad hoc duties.

19 - These are often patients with impatience, demanding a quick solution. As a GP you want to take away the agitation (and sometimes the 'demanding' behaviour) and quickly offer a bit of relief and structure through explanation and medication. The procedure through trial participation is too exhaustive.

- As a GP, I don't want 'this' patient to be randomised in the control arm; because I already changed my prescribing patterns a few years ago, so the treatment arm is how I currently treat my patients.

20 In the acute phase you already act on your findings; so if morphine is needed, you have already prescribed this before the trial comes into focus.

21 - This is a proactive study, therefore I have to remember it at the moment itself.

- Usually the treatment of choice is the result of a - sort of - negotiation between patient and GP.

- Often patients already used paracetamol, or react like: 'Paracetamol?', and they feel unheard.

22 - Myths about opioids by patients.

- Patients want to start with medication directly.

- In our general practice there's often a language barrier which increases difficulty when it comes to explaining the trial and answering questionnaires.

- Incidence

- Eligibility criteria

- Priority setting

- Shared decision making

- Delay

- Delay

- Shared decision making

- Forgetfulness

- Shared decision making

- Eligibility criteria

- Delay

Table 1. Summary of responses of 22/116 (19\%) GPs

laws, and organising safe digital working systems; GPs spend a considerable amount of time on managing these processes. This may have (indirectly) influenced the present research process; both the willingness and/or time available to participate in research may decrease subsequently.

Reasons for insufficient recruitment

GPs: $n$

$(\%)^{a}$

Shared decision-making process: (strong) patient preference and/or GP preference for specific medication, diagnostics, randomisation 11 (50) outcome

Low incidence rate

$6(27)$

Restrictive eligibility criteria

$6(27)$

Priority setting: too many other responsibilities/administrative burden, time consuming/time pressure

$6(27)$

No further delay wished

$5(23)$

Forgetfulness

$5(23)$

Lack of motivation

$4(18)$

Other reasons

$4(18)$

${ }^{a}$ More than one answer was possible. 


\section{Discussion}

\section{Summary}

This study described the rationale behind a prematurely discontinued RCT and identified several barriers that could have contributed to the low inclusion rate. The main study-related factors were the strict eligibility criteria and the trial design, aiming to enrol incident cases with an acute condition. The main external factors were societal developments and changes in patient populations, prescription habits, and the doctor-patient relationship. Research is needed to expand evidence for guidelines in the diagnosis and treatment of diseases within primary care. This article describes a trial that was not successful, to enable other researchers and GPs to learn from this experience.

\section{Strengths and limitations}

This study's main limitation was that the protocol seemed too complex to fit well into the daily clinic, for example resulting in a problem for patients with an acute need for pain medication due to the informed consent procedure. It was not possible to determine further strengths and limitations as the trial was prematurely discontinued.

\section{Comparison with existing literature}

This experience is not new: achieving sufficient patient recruitment in clinical trials in general practice is an ongoing problem. ${ }^{24-26}$ Many prospective studies depend on GPs to recruit patients. However, for various understandable reasons, there is a discrepancy between the number of GPs that agree to participate and the number that actually recruit patients, even though they thought it was feasible in practice. ${ }^{27,28}$ This is why training of clinical staff — such as GPs, GP assistants, research nurses - is vital to improve patient recruitment, as mentioned by Donovan et al. ${ }^{29,30}$ Their research shows increases in trial participants after staff training programmes and individual tailored feedback and support. Bower et al suggests to increase public and professional engagement with research to improve participation by both clinicians and patients. ${ }^{31}$

Literature research in this field suggests that, in general, the recruitment rate (even in successful trials) is lower than anticipated. ${ }^{32}$ Apparently researchers are often too optimistic about the number of eligible patients, and the GPs' time and ability to recruit patients. ${ }^{33}$ According to Lasagna's Law, they overestimate the incidence of patient availability. ${ }^{24}$ This addresses the difficulty of predicting numbers by researchers, especially in studies with complex eligibility criteria and in studies that require incident cases of acute conditions. ${ }^{34}$ For future research, a pilot study should be performed (if financially feasible) in which the inclusion/exclusion criteria are tested before going full scale. ${ }^{24,35}$ Alternatively, as suggested by Haidich et al, patient enrolment patterns during the first 2 months can be analysed and will often predict the eventual ability of a trial to attain its target sample size. ${ }^{36}$

Some determinants are associated with a higher risk of recruitment failure; for example, restrictive eligibility criteria which is often a result of the difficult balance between diminishing risks for individual patients and maintaining the generalisability of trial results, and studies interfering with the GP's decision-making process; that is, when recruiting patients has a direct impact on usual care after the randomisation process. ${ }^{33}$ A systematic review highlights the same main barriers for clinicians and patients experienced in the current study, including time constraints, potential impact on the doctor-patient relationship, concern for the patient, loss of professional autonomy, patient preferences, and/or difficulty with the consent procedure. ${ }^{37}$ Treweek et al suggests that a financial incentive for patients could have been a promising strategy to increase patient recruitment, next to an open trial design and the telephone reminders already used in this trial. ${ }^{38}$ The PRECIS wheel is a tool developed by Thorpe et al that can be used by researchers to help them designing a trial that is consistent with their purpose. ${ }^{39}$ Reflecting on this, because of the nature of the disease (sciatica) and the strength of the medication this trial was testing (morphine), the in- and exclusion criteria of the current trial were rather narrow, while the trial design itself was pragmatic. Comorbidities that were sometimes common, where reasons for exclusion in this trial. From that perspective, this trial appeared to be less pragmatic than orginally aimed for. 


\section{Implications for research and practice}

From the GPs' viewpoint there was a contradiction between practising research or practicing medicine in the consultation room. As researchers, we should aim to close this gap by designing simple and convenient protocols that facilitate participation in research during busy consultation schedules. Training and support should be offered when needed. ${ }^{40}$ If possible, researchers should prevent too much interference in regular practice and create a format that fits in the daily routine tasks of GPs. Referring a patient to research should be as easy as referring someone to a specialist or paramedical discipline: preferably, one 'click' away, no paperwork involved, and using existing digital referral systems.

The introduction of practice nurses for both somatic and psychological care paved the way for task delegation in chronic care and psychosocial support. Regarding evidence-based medicine in primary care, it is worthwhile to investigate the possibilities of involving research nurses in general practice to make research a part of daily practice. However, the applicability of dedicated research nurses in general practice might be limited, both for logistic reasons (wide variation in staffing models worldwide), financial (who pays) and practical reasons (for example, access to patient information might be limited for research nurses because of privacy laws). Aiming for more involvement of GP assistants in the recruitment process is a more pragmatic and achievable solution. Compensation for the practice for the additional time and expenses related to the research in that case will be a point of discussion. Another possibility is to combine care and research into an integrated service/ research clinic, as is more often seen in the UK. ${ }^{31,41,42}$

In summary, several recommendations can be made in the aim to try and avoid/prevent recruitment problems:

- if financially possible, perform a pilot study to investigate the feasibility of recruitment and examine potential threats before going full scale; and

- use of dedicated research staff in larger general practices, or involving trained GP assistants in an early stage of the research in smaller practices, might be an effective way to support GPs to outsource eligibility screening and study entry.

For future research, recommended topics are:

- to address important research questions with clear clinical relevance and of daily interest for GPs and their patients. ${ }^{31}$ The protocol and data collection should be straightforward, to have the least disruptive impact on regular care and doctor-patient interaction; ${ }^{43}$ and

- to have no or restricted impact on usual care delivery during consultations and only register how care is delivered, to minimise the demands made on GPs and their patients.

To conclude, although multiple factors played a role in the unsuccessful recruitment for this trial, it remains unknown which of the determinants played the most important role.

\section{Funding}

This study was funded by the Netherlands Organization for Health Research and Development (reference 80-83600-98-20013) and a programme grant from the Dutch Arthritis Foundation.

\section{Ethical approval}

The study protocol was approved by the Medical Ethical Committee of Erasmus MC (NL49507.078.14). The trial is registered at the Dutch Trial Registry (NTR5120, 26 March 2015. Protocol version: 3 January, 2015).

Trial registration

Dutch Trial Registry, NTR5120.

\section{Acknowledgements}

The authors thank the participating patients and GPs for their cooperation; and Dr MA Koopmanschap, Department of Health Policy and Management/iMTA, for his contribution during the drafting of the research protocol.

Provenance

Freely submitted; externally peer reviewed. 


\section{References}

1. Schäfer WL, Boerma WG, Spreeuwenberg P, et al. Two decades of change in European general practice service profiles: conditions associated with the developments in 28 countries between 1993 and 2012. Scand J Prim Health Care 2016; 34(1): 97-110. doi: 10.3109/02813432.2015.1132887

2. Groenewegen PP, Hutten JB, van der Velden K. List size, composition of practice and general practitioners' workload in The Netherlands. Soc Sci Med 1992; 34(3): 263-270. doi: 10.1016/0277-9536(92)90268-U

3. van den Berg MKE, De Bakker D, Van der Zee J. The workload of general practitioners in the Netherlands: 1987 and 2001. In: Westert GPJ L, Schellevis FG (eds) Morbidity, performance and quality in primary care. Dutch general practice on stage. Oxford: Radcliffe Publishing. 2006.

4. Schäfer WGP, van den Berg M. The workload of general practitioners; the Netherlands in an international perspective. [In Dutch]. Utrecht: NIVEL, 2016.

5. Schäfer WLA vdBM, Groenewegen PP. The workload of GPs in international perspective. [In Dutch]. Huisarts en Wetenschap 2016; 59(3): 94-101.

6. Brænd $A M$, Jensen $K B$, Klovning $A$, et al. Clinical drug trials in general practice: a 10-year overview of protocols. Trials 2013; 14: 162-162. doi: 10.1186/1745-6215-14-162

7. Hummers-Pradier E, Bleidorn J, Schmiemann G, et al. General practice-based clinical trials in Germany - a problem analysis. Trials 2012; 13: 205-205. doi: 10.1186/1745-6215-13-205

8. Fransen GA, van Marrewijk CJ, Mujakovic S, et al. Pragmatic trials in primary care. Methodological challenges and solutions demonstrated by the DIAMOND-study. BMC Med Res Methodol 2007; 7(1): 1-11. doi: 10.1186/1471-2288-7-16

9. Sully BG, Julious SA, Nicholl J. A reinvestigation of recruitment to randomised, controlled, multicenter trials: a review of trials funded by two UK funding agencies. Trials 2013; 14: 14. doi: 10.1186/1745-6215-14-166

10. Gágyor I, Bleidorn J, Wegscheider K, et al. Practices, patients and (im)perfect data-feasibility of a randomised controlled clinical drug trial in German general practices. Trials 2011; 12: 91-91. doi: 10.1186/ 1745-6215-12-91

11. Schaafstra ASW, Bons SCS, Borg M, et al. Dutch College of General Practitioners' guideline: lumbosacral radicular syndrome [In Dutch]. Huisarts en Wetenschap 2015; 58(6): 308-320.

12. Pinto RZ, Maher CG, Ferreira ML, et al. Drugs for relief of pain in patients with sciatica: systematic review and meta-analysis. BMJ 2012; 344: e497. doi: 10.1136/bmj.e497

13. Register DT. Step-down versus step-up analgesics in patients with (sub)acute sciatica in primary care (NTR5120). http://www.trialregister.nl/trialreg/admin/rctview.asp?TC=5120 (accessed 11 Jul 2017).

14. Luijsterburg PA, Verhagen AP, Ostelo RW, et al. Physical therapy plus general practitioners' care versus general practitioners' care alone for sciatica: a randomised clinical trial with a 12-month follow-up. Eur Spine J 2008; 17(4): 509-517. doi: 10.1007/s00586-007-0569-6

15. Health Council of the Netherlands. Reports 1999. A2000/01. 2000. https://www.gezondheidsraad.nl/sites/ default/files/A0001E.pdf (accessed 27 Jul 2017)

16. NIVEL Primary Care Database. Incidence and prevalence numbers in general practice. https://www.nivel.nl/ nl/NZR/incidenties-en-prevalenties (accessed 28 Jul 2017).

17. International Narcotics Control Board. Narcotic Drugs Technical Report: estimated world requirements for 2013 - statistics for 2011. New York, NY: United Nations, 2013.

18. Fischer B, Jones W, Rehm J. Trends and changes in prescription opioid analgesic dispensing in Canada 2005-2012: an update with a focus on recent interventions. BMC Health Serv Res 2014; 14: 90. doi: 10.1186/ 1472-6963-14-90

19. Manchikanti L, Helm S, Fellows B, et al. Opioid epidemic in the United States. Pain Physician 2012; 15(3 Suppl): ES9-38.

20. Smolina K, Gladstone E, Morgan SG. Determinants of trends in prescription opioid use in British Columbia, Canada, 2005-2013. Pharmacoepidemiol Drug Saf 2016; 25(5): 553-559. doi: 10.1002/pds.3989

21. Karanges EA, Blanch B, Buckley NA, et al. Twenty-five years of prescription opioid use in Australia: a wholeof-population analysis using pharmaceutical claims. Br J Clin Pharmacol 2016; 82(1): 255-267. doi: 10.1111/ bcp. 12937

22. Berger JT. Redefining the domains of decision making by physician and patient. Int J Clin Pract 2011; 65(8): 828-830. doi: 10.1111/j.1742-1241.2011.02708.x

23. Barry MJ, Edgman-Levitan S. Shared decision making-pinnacle of patient-centered care. $N$ Engl J Med 2012; 366(9): 780-781. doi: 10.1056/NEJMp1109283

24. van der Wouden JC, Blankenstein AH, Huibers MJ, et al. Survey among 78 studies showed that Lasagna's law holds in Dutch primary care research. J Clin Epidemiol 2007; 60(8): 819-824. doi: 10.1016/j.jclinepi. 2006.11.010

25. van der Windt DA, Koes BW, van Aarst $M$, et al. Practical aspects of conducting a pragmatic randomised trial in primary care: patient recruitment and outcome assessment. Br J Gen Pract 2000; 50(454): 371-374.

26. Montfoort I, Frens MA, Koes BW, et al. Tragedy of conducting a clinical trial; generic alert system needed. $J$ Clin Epidemiol 2008; 61(5): 415-418. doi: 10.1016/j.jclinepi.2007.06.004

27. Tognoni G, Alli C, Avanzini F, et al. Randomised clinical trials in general practice: lessons from a failure. BMJ 1991; 303(6808): 969-971. doi: 10.1136/bmj.303.6808.969

28. Peto V, Coulter A, Bond A. Factors affecting general practitioners' recruitment of patients into a prospective study. Fam Pract 1993; 10(2): 207-211. doi: 10.1093/fampra/10.2.207 
29. Donovan JL, Lane JA, Peters TJ, et al. Development of a complex intervention improved randomization and informed consent in a randomized controlled trial. J Clin Epidemiol 2009; 62(1): 29-36. doi: 10.1016/j. jclinepi.2008.02.010

30. Donovan JL, de Salis I, Toerien $\mathrm{M}$, et al. The intellectual challenges and emotional consequences of equipoise contributed to the fragility of recruitment in six randomized controlled trials. J Clin Epidemiol 2014; 67(8): 912-920. doi: 10.1016/j.jclinepi.2014.03.010

31. Bower $P$, Wallace $P$, Ward $E$, et al. Improving recruitment to health research in primary care. Fam Pract 2009; 26(5): 391-397. doi: 10.1093/fampra/cmp037

32. Bower $\mathrm{P}$, Wilson $\mathrm{S}$, Mathers $\mathrm{N}$. Short report: how often do UK primary care trials face recruitment delays? Family Practice 2007; 24(6): 601-603. doi: 10.1093/fampra/cmm051

33. Foy R, Parry J, Duggan A, et al. How evidence based are recruitment strategies to randomized controlled trials in primary care? Experience from seven studies. Fam Pract 2003; 20(1): 83-92. doi: 10.1093/fampra/20. 1.83

34. White D, Hind D. Projection of participant recruitment to primary care research: a qualitative study. Trials 2015; 16: 473. doi: 10.1186/s13063-015-1002-9

35. Stein MA, Shaffer M, Echo-Hawk A, et al. Research START: a multimethod study of barriers and accelerators of recruiting research participants. Clin Trans/ Sci 2015; 8(6): 647-654. doi: 10.1111/cts.12351

36. Haidich $A B$, loannidis JP. Patterns of patient enrollment in randomized controlled trials. $J$ Clin Epidemiol 2001; 54(9): 877-883. doi: 10.1016/S0895-4356(01)00353-5

37. Ross S, Grant A, Counsell C, et al. Barriers to participation in randomised controlled trials: a systematic review. J Clin Epidemiol 1999; 52(12): 1143-1156.

38. Treweek $S$, Lockhart $P$, Pitkethly $M$, et al. Methods to improve recruitment to randomised controlled trials: Cochrane systematic review and meta-analysis. BMJ Open 2013; 3(2): e002360. doi: 10.1136/bmjopen2012-002360

39. Thorpe $K E$, Zwarenstein $M$, Oxman $A D$, et al. A pragmatic-explanatory continuum indicator summary (PRECIS): a tool to help trial designers. J Clin Epidemiol 2009; 62(5): 464-475. doi: 10.1016/j.jclinepi.2008. 12.011

40. Donovan JL, Paramasivan $\mathrm{S}$, de Salis $\mathrm{I}$, et al. Clear obstacles and hidden challenges: understanding recruiter perspectives in six pragmatic randomised controlled trials. Trials 2014; 15: 5-5. doi: 10.1186/1745-6215-15-5

41. Konstantinou K, Beardmore R, Dunn KM, et al. Clinical course, characteristics and prognostic indicators in patients presenting with back and leg pain in primary care. The ATLAS study protocol. BMC Musculoskelet Disord 2012; 13: 4-4. doi: 10.1186/1471-2474-13-4

42. Shaw S, Macfarlane F, Greaves C, et al. Developing research management and governance capacity in primary care organizations: transferable learning from a qualitative evaluation of UK pilot sites. Fam Pract 2004; 21(1): 92-98. doi: 10.1093/fampra/cmh120

43. Pringle $M$, Churchill R. Randomised controlled trials in general practice. BMJ 1995; 311(7017): 1382-1383. doi: 10.1136/bmj.311.7017.1382 